\title{
An Efficient Dynamic Analysis of Planar Arches
}

\section{Beytullah Temel', Timuçin Alp Aslan², Ahmad Reshad Noori ${ }^{3^{*}}$}

\author{
1,2,3 Department of Civil Engineering, Cukurova University, Adana, Turkey
}

\begin{abstract}
The aim of this study is to investigate the curved rods subjected to dynamic loads with variable geometric properties along the axis theoretically. The transient analysis of the planar rods under the various in-plane dynamic loads has been analyzed in the Laplace domain. The obtained canonical form of the first order ordinary differential equations has been solved by Complementary Functions Method (CFM) in the transformed domain. The fifth-order Runge-Kutta method has been applied to the solution of the obtained equations. The materials of the structural elements are assumed to be homogeneous, isotropic, and elastic. The solutions obtained are transformed to the time domain using the modified Durbin's inverse numerical Laplace transformed method. For the suggested models, a computer program is coded in Fortran for the dynamic analysis of the planar curved structural elements. Verification of the computer program is performed by comparing the results of the present methods with the other numerical methods available in the literature. The procedures have been proved to be highly accurate and efficient compared to various other numerical methods.
\end{abstract}

Keywords: Two-Point Boundary Value Problems, Complementary Functions Method (CFM), Inverse Laplace Transforms, Planar Arches.

\section{INTRODUCTION}

Rods are very important structural elements in several engineering fields such as automotive, mechanical and civil engineering. Generally, curved rods are used as curved structural elements in bridges, aqueducts, stairs, vaulted roofs, and arches. Due to the importance of those engineering structures, it is significant to determine the behavior of curved rods under both static and dynamic loads.

Many researchers have investigated the static and dynamic behavior of rods. Among those, Haktanır [1] investigated the static behavior of in-plane rods, made up of isotropic-elastic material, by stiffness matrix method based on Complementary Functions Method (CFM). The Runge-Kutta fourth-order method has been used for the solution of the obtained equations. Bayhan [2] studied the static behavior of planar frames with members of circular axes with the aid of both transfer and stiffness matrix methods. Bozkurt [3] used Complementary Functions Method (CFM) for the bending analysis of circular planar systems, helicoidal stair cases, axisymmetric shell structures, and cylindrical vault structures under static loadings. Yildirim et. al.[4] investigated the static analysis of compound planar frames with members of linear and circular axes by stiffness matrix method. The stiffness matrixes and force vectors of the frame members with circular axes, under planar loads and perpendicular loads to plane, were calculated by transform matrix method precisely.

The dynamic behavior of cylindrical helical rods made of isotropic, anisotropic and elastic, viscoelastic materials under time dependent loads is investigated by Çalım [5] in the Laplace domain theoretically. In the solutions, Kelvin model was employed. The obtained solutions transformed to the time domain by using a proper inverse Laplace transform method. Kıraç [6] examined the dynamic behavior of composite straight rods subjected to time-dependent loads in the Laplace domain theoretically. The free vibration was considered as a special case of forced vibration. In the formulations, the effect of the rotary inertia, axial and shear deformations were taken into account. The ordinary differential equations obtained in canonical form in the Laplace domain were solved numerically by the Complementary Functions Method to calculate the dynamic stiffness matrix. Aktan [7] studied the free vibration of in-plane circular beams. The Timoshenko beam theory was used and the effect of the rotary inertia was considered. Çoban [8] investigated the dynamic analysis of curved beams using mixed finite element method with Gâteaux derivative. Akkurt [9] investigated the dynamic behavior of straight and circular rods resting on elastic foundation in the Laplace domain theoretically. Karaca [10] studied the static and dynamic analysis of circular Timoshenko rods which are loaded in-plane and out-of-plane, theoretically.

\footnotetext{
*Corresponding authour

Email: a.reshadnoori@yahoo.com (A.R.Noori)

$\S$ This paper was presented in the IMSEC-2016
} 
The longitudinal vibrations of elastic bars were investigated using the Gâteaux differential method and the mixed finite element method by Ecer [11].

Literature survey shows that many studies dealing with static and free vibration analysis of curved rods have been made and several methods have been investigated by researchers. However, there are limited studies in the literature addressing to the solutions of in-plane curved rods subjected to time variable dynamic loads by Complementary Functions Method in the Laplace domain. In present paper the forced vibration of in-plane curved rods has been analyzed under various types of dynamic loads by Laplace transform method and Complementary Functions Method for the first time. The CFM is a numerical solution method which transforms a two point boundary value problem to a system of initial value problems. For the solution of initial value problems fifth order Runge-Kutta is applied in this study. The application of Laplace Transform, with respect to time, to partial differential equations, converts them to ordinary differential equations in the transformed domain. Thus the numerical solution of partial differential equations in the Laplace domain can be done easily. To transform the obtained solutions in the Laplace domain to the time domain an efficient inverse Laplace transform method has been used. The results of the present method are compared with those solutions given in the literature and it has been seen that he results obtained in this study are found to be in good agreement with those available in the literature.

\section{EQUATIONS OF MOTIONS}

The time and location depended partial differential equations of in-plane rods under dynamic loads are given as follows

$$
\begin{aligned}
& \frac{\partial U_{t}}{\partial \varphi}=U_{n}+r \frac{T_{t}}{E A(\varphi)} \\
& \frac{\partial U_{n}}{\partial \varphi}=-U_{t}+r \Omega_{b}+r \frac{T_{n} \alpha_{n}}{G A(\varphi)} \\
& \frac{\partial \Omega_{b}}{\partial \varphi}=r \frac{M_{b}}{E I_{b}(\varphi)} \\
& \frac{\partial T_{t}}{\partial \varphi}=r \rho A(\varphi) \frac{\partial^{2} U_{t}}{\partial t^{2}}+T_{n}-r p_{t} \\
& \frac{\partial T_{n}}{\partial \varphi}=r \rho A(\varphi) \frac{\partial^{2} U_{n}}{\partial t^{2}}-T_{t}-r p_{n} \\
& \frac{\partial M_{b}}{\partial \varphi}=r \rho I_{b}(\varphi) \frac{\partial^{2} \Omega_{b}}{\partial t^{2}}-r T_{n}-r m_{b}
\end{aligned}
$$

Here, $E, \rho, h(\phi), A(\phi), I_{b}(\phi)$ and $\alpha_{n}$ indicate the modulus of elasticity, mass density, section area, the moment of inertia and shear correction factor respectively. The unknown column matrix, $\{Y(\phi, t)\}$, for the forced vibration of in-plane loaded rods is given as:

$$
\{Y(\phi, t)\}=\left\{U_{t}, U_{n}, \Omega_{b}, T_{t}, T_{n}, M_{b}\right\}^{T}
$$

Applying the Laplace transform to equations (1-6), converts these partial differential equations to variable-coefficient ordinary differential equations. Thereby, the governing ordinary differential equations of the dynamic behavior of in-plane loaded curved rods can be obtained in the Laplace domain as follows

$$
\begin{aligned}
& \frac{d \bar{U}_{t}}{d \varphi}=\bar{U}_{n}+r \frac{\bar{T}_{t}}{E A(\varphi)} \\
& \frac{d \bar{U}_{n}}{d \varphi}=-\bar{U}_{t}+r \bar{\Omega}_{b}+r \frac{\bar{T}_{n} \alpha_{n}}{G A(\varphi)} \\
& \frac{d \bar{\Omega}_{b}}{d \varphi}=r \frac{\bar{M}_{b}}{E I_{b}(\varphi)}
\end{aligned}
$$




$$
\begin{aligned}
& \frac{d \bar{T}_{t}}{d \varphi}=r s^{2} \rho A(\varphi) \bar{U}_{t}+\bar{T}_{n}-r \bar{p}_{t} \\
& \frac{d \bar{T}_{n}}{d \varphi}=r s^{2} \rho A(\varphi) \bar{U}_{n}-\bar{T}_{t}-r \bar{p}_{n} \\
& \frac{d \bar{M}_{b}}{d \varphi}=r s^{2} \rho I_{b}(\varphi) \bar{\Omega}_{b}-r \bar{T}_{n}-r \bar{m}_{b}
\end{aligned}
$$

Where the terms shown by indicates the Laplace transform of the quantities.

The Laplace transformation of axial, flexural and mass moment of inertia are given as:

$$
\begin{aligned}
& L\left[\rho A(\varphi) \frac{\partial^{2} U_{t}}{\partial t^{2}}\right]=\rho A(\varphi)\left[s^{2} \bar{U}_{t}-s U_{t}(\varphi, 0)-\frac{\partial U_{t}(\varphi, 0)}{\partial t}\right] \\
& L\left[\rho A(\varphi) \frac{\partial^{2} U_{n}}{\partial t^{2}}\right]=\rho A(\varphi)\left[s^{2} \bar{U}_{n}-s U_{n}(\varphi, 0)-\frac{\partial U_{n}(\varphi, 0)}{\partial t}\right] \\
& L\left[\rho I_{b}(\varphi) \frac{\partial^{2} \Omega_{b}}{\partial t^{2}}\right]=\rho I_{b}(\varphi)\left[s^{2} \bar{\Omega}_{b}-s \Omega_{b}(\varphi, 0)-\frac{\partial \Omega_{b}(\varphi, 0)}{\partial t}\right]
\end{aligned}
$$

The second and third terms on the right-hand side of the equation (14) are the initial conditions given for $t=0$; in present study those terms are assumed to be zero.

\section{COMPLEMENTARY FUNCTIONS METHOD}

The matrix notation of the ordinary differential equations (8-13) obtained in the Laplace domain is given below;

$$
\frac{d\{\bar{Y}(\phi, s)\}}{d \phi}=[\bar{A}(\phi, s)]\{\bar{Y}(\phi, s)\}+\{\bar{F}(\phi, s)\}
$$

Here is independent variable and $z$ is the Laplace transform parameter. The state vector for in-plane curved rods is given by equation (16).

$$
\{\bar{Y}(\phi, s)\}=\left\{\bar{U}_{t}(\phi, s), \bar{U}_{n}(\phi, s), \bar{\Omega}_{b}(\phi, s), \bar{T}_{t}(\phi, s), \bar{T}_{n}(\phi, s), \bar{M}_{b}(\phi, s)\right\}^{T}
$$

The complementary functions method is based on the principle of the solution of equation (15) with the help of the initial conditions. This method is basically the reduction of two-point boundary value problems to initial-value problems. The general solution of Eq. (15), is given by

$$
\{Y(\phi, s)\}=\sum_{m=1}^{n} C_{m}\left[\bar{U}^{(m)}(\phi, s)\right]+\{\bar{V}(\phi, s)\}
$$

Where $\left[\bar{U}^{(m)}(\phi, s)\right]$ is the complementary solution such that its $m^{\text {th }}$ component is equal to 1 , whereas all the others are 0. $\{\bar{V}(\phi, s)\}$ is the inhomogeneous solution with all 0 initial conditions, the integration constants $C_{m}$ will be determined from the boundary conditions at both ends.

The results, obtained in the Laplace domain, are transformed to the time domain with the help of modified Durbin's numerical inverse Laplace transform method (Durbin, 1974, Temel et al., 2004).

\section{NUMERICAL EXAMPLES AND DISCUSSION}

A pin-ended isotropic parabolic arch, shown in figure 1.a, is now considered under a point dynamic load, applied to its midpoint. Material properties density, $\rho=7850 \times 10^{-6} \mathrm{kgf} / \mathrm{cm}^{3}$, Poisson's ratio, $v=0.3$, and modulus of elasticity, $\rho=7850 \times 10^{-6} \mathrm{kgf} / \mathrm{cm}^{3}$. Two types of dynamic point loads, shown in figure $1 . \mathrm{b}$, with the amplitude $p_{0}=1 \mathrm{~kg}$ are applied on the plate.

The axis line equation of the parabolic arch in the rectangular coordinate system shown in figure 1.a. and changes of the radius of curvature or " $r$ " along the axis can be expressed as follows respectively. 


$$
y=\frac{4 \not^{2}}{L^{2}}, r=\frac{r_{0}}{\cos ^{3} \phi}
$$

Where $r_{0}$ indicates the radius of curvature of Point $\mathrm{O}$.

$$
r_{0}=\frac{L^{2}}{8 f}
$$

Here it is considered that $I_{b}(\phi) \operatorname{Cos} \phi=I_{b}(0)$ is constant and hence the flexural rigidity and cross section of arch is variable. So the flexural rigidity is taken as

$$
D_{b}=\frac{E_{b}(0)}{\operatorname{Cos} \phi}, A(\phi)=\frac{A(0)}{\operatorname{Cos} \phi}
$$

The boundary conditions of pinned-end and symmetric point are given as follows

$$
\phi=0 \rightarrow\left\{\begin{array}{c}
U_{t}=0 \\
\Omega_{b}=0 \\
T_{n}=-p / 2
\end{array} \quad \phi=\phi_{0} \rightarrow\left\{\begin{array}{l}
U_{t}=0 \\
U_{n}=0 \\
M_{b}=0
\end{array}\right.\right.
$$

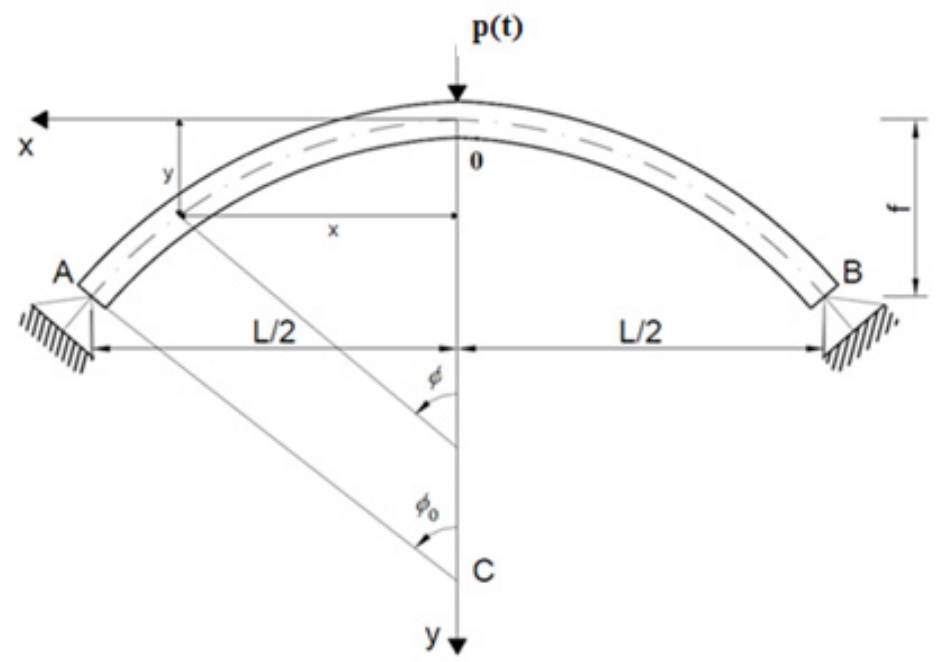

(a)
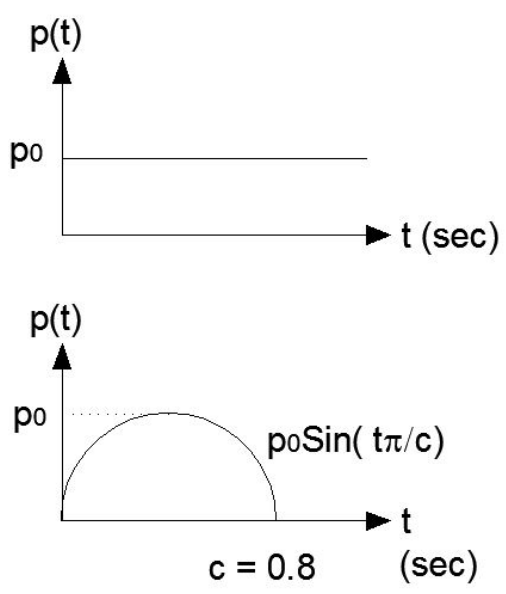

(b)

Figure 1: (a) Pin-ended parabolic arch; (b) Dynamic loads;

The equations (8-13) given in canonical form are solved numerically in the Laplace domain by CFM. The results are compared with those obtained from ANSYS finite element software, which uses the Newmark time integration method to solve the equations of motion. Comparisons are shown in the graphics. In this problem the effect of shear deformation is taken into account.

The geometric properties of the parabolic arch are as follows.

$A(0)=1 \mathrm{~cm}^{2} ; I_{b}(0)=0.0833 \mathrm{~cm}^{4} ; L=200 \mathrm{~cm}, r_{0}=100 \mathrm{~cm} ; f=50 \mathrm{~cm} ; p_{0}=1 \mathrm{~kg} ; \phi_{0}=45^{\alpha} ; \alpha_{n}=1$

The vertical displacement of the midpoint of the parabolic arch under a point step load obtained by present method is presented in Fig. 2. The problem has been solved for various time increments as transform parameters ( $d t=0.08$ sec., $N$ $=64),(d t=0.04 \mathrm{sec} ., N=256),(d t=0.02 \mathrm{sec} ., N=256)$ and $(d t=0.01 \mathrm{sec} ., N=512)$. It is apparent that results obtained for a coarse time increment along with fewer Laplace transform parameters overlap the results obtained with finer increments and higher parameters. This indicates the efficiency of the present method. 


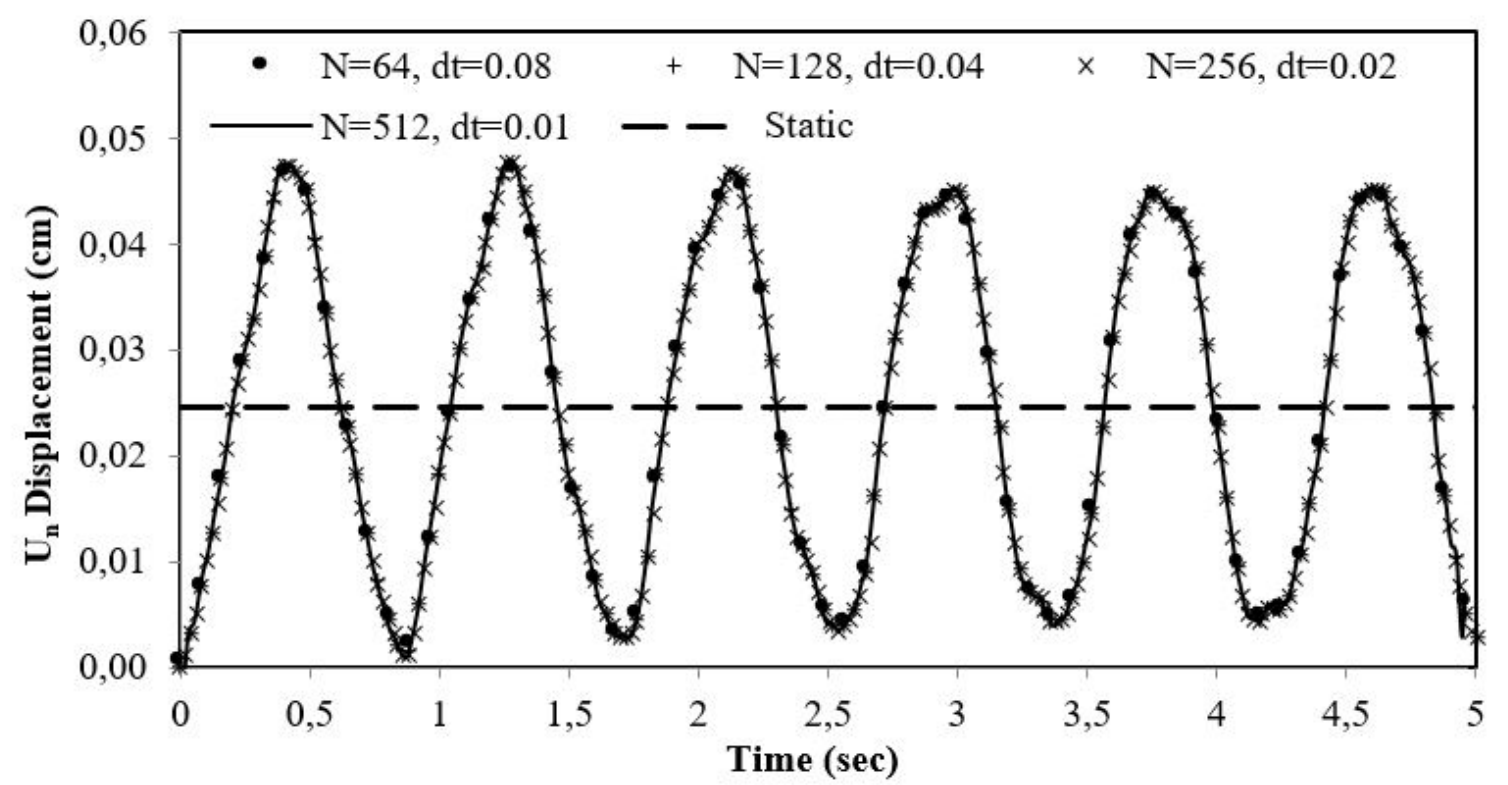

Figure 2: Vertical displacement of the midpoint of the parabolic arch under a point step load

The parabolic arch is divided into 40 uniaxial elements in ANSYS while the cross section of the arch is considered to be constant. In respect to this the flexural rigidity and cross section of the curved rods is taken as $D_{b}=E I_{b}(0)$ and $A(\phi)=$ $A(0)$ respectively in CFM method for all solutions of step loading. The comparison of proposed method and Newmark method for various time increments and constant cross section given in Figs. 3 and 4 show the $U_{n}$ vertical displacement and $M_{b}$ bending moment at the midpoint of the arch for a step load respectively. It can be seen that time increments of $0.01 \mathrm{sec}$. and finer had to be considered for consistent results in ANSYS. An exact match is obtained by using a coarse time increment of $0.08 \mathrm{sec}$. in the present method as opposed to much finer increment of $0.01 \mathrm{sec}$. in Newmark method. The proposed method leads to considerable saving in computation time.

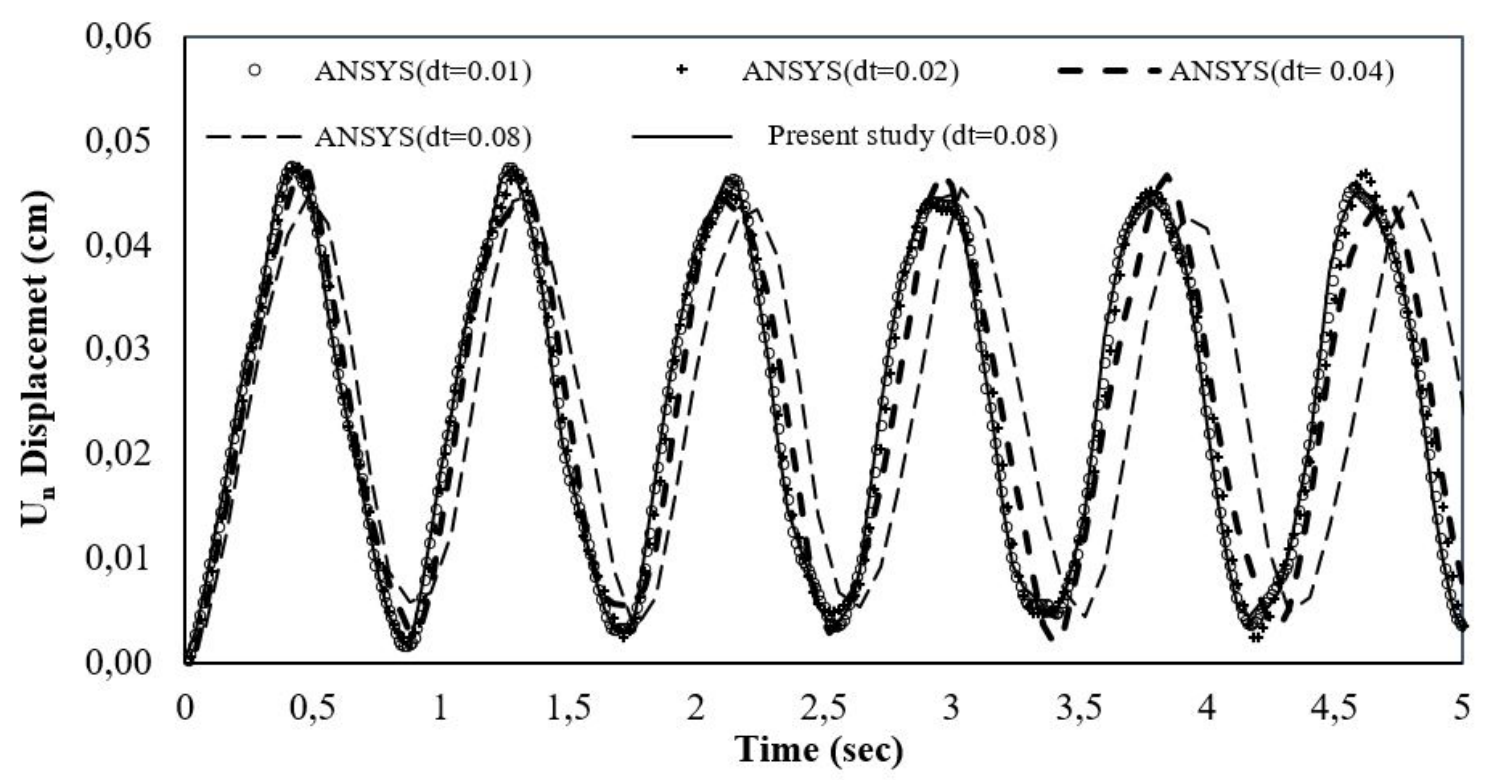

Figure 3: Comparison of $U_{n}$ vertical displacement versus time at the arch midpoint for step load. 


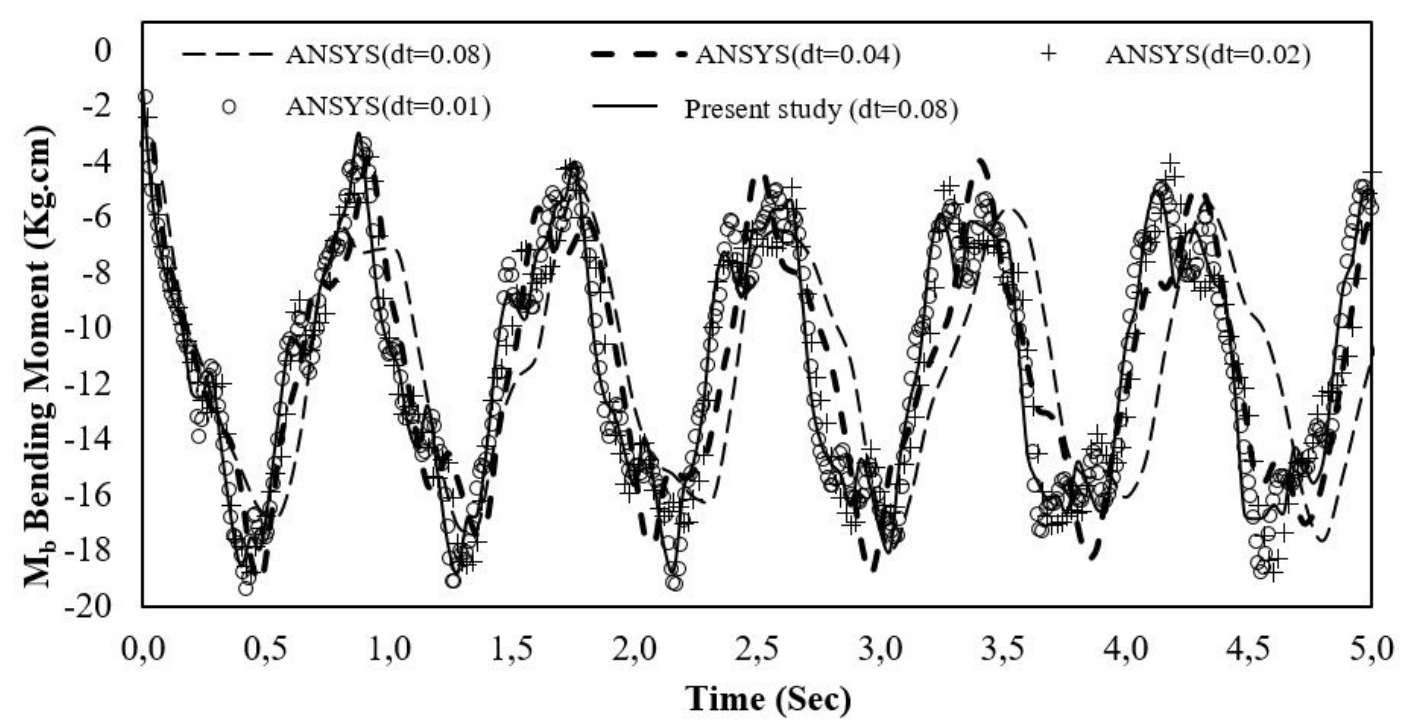

Figure 4: Comparison of $\mathrm{M}_{\mathrm{b}}$ bending moment versus time at the arch midpoint for step load.

The cross section of the parabolic arch is considered to be variable under sinusoidal load. The vertical displacement Un and $\mathrm{Mb}$ moment of bending at the arch midpoint under a sinusoidal load for elastic material are shown in Figs.5 and 6, respectively. The figures include the elastic-dynamic case.

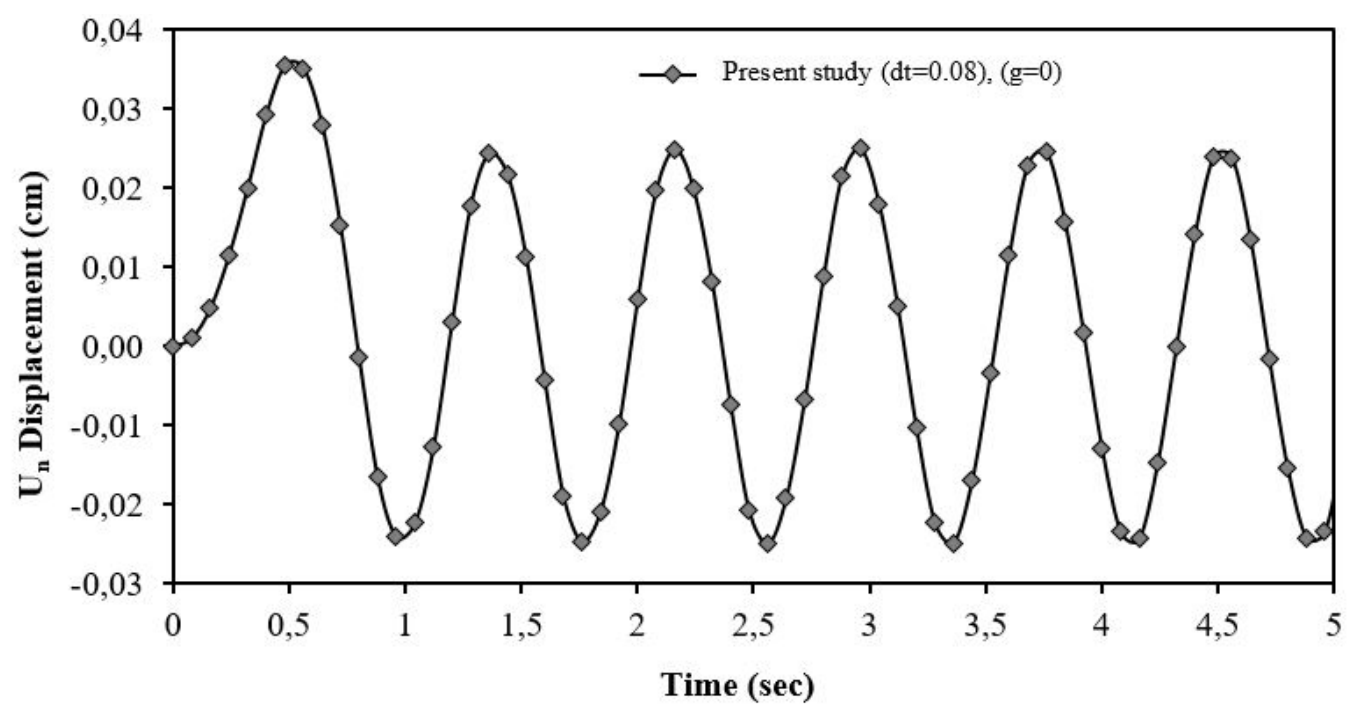

Figure 5: $\mathrm{U}_{\mathrm{n}}$ vertical displacement versus time at the arch midpoint for sinusoidal load.

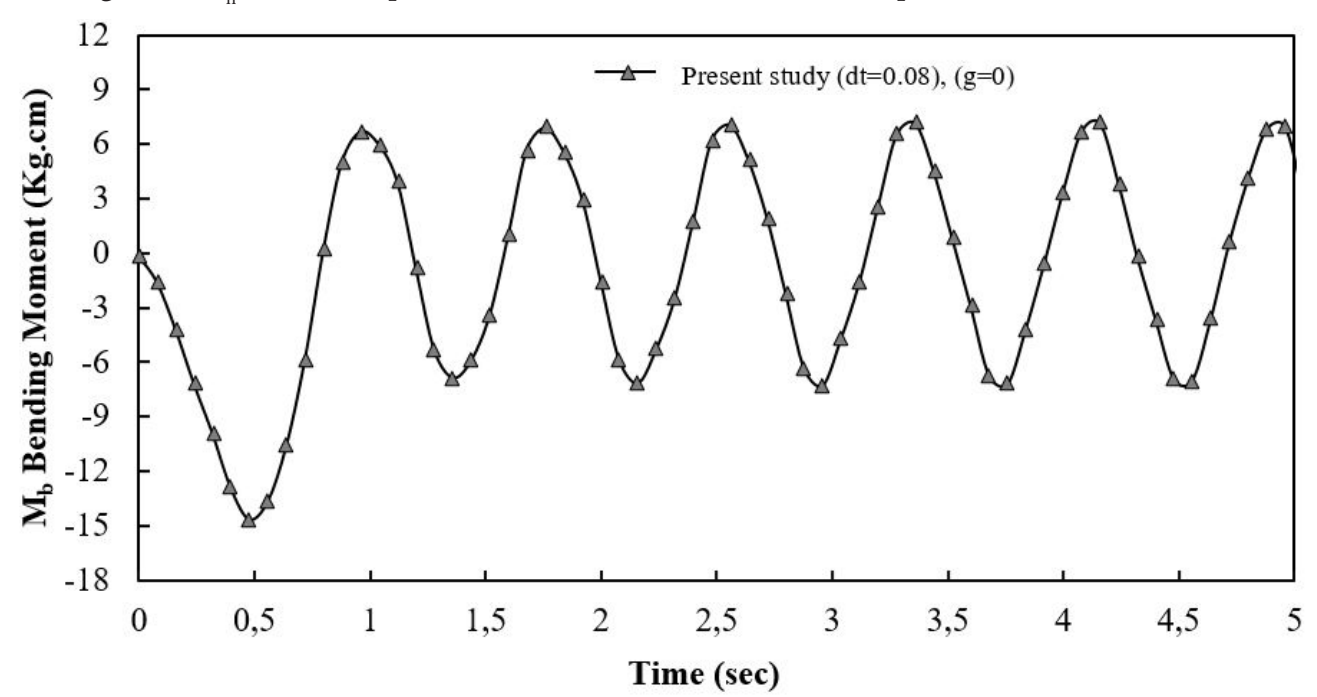

Figure 6: $\mathrm{M}_{\mathrm{b}}$ Moment of bending versus time at the arch midpoint for sinusoidal load. 


\section{CONCLUSION}

In this paper, the forced vibration of planar curved rods is investigated under various types of dynamic loads. The dynamic behavior of such structures is examined in Laplace domain by CFM. Fifth order Runge-Kutta algorithm is used for the solution of the initial value problems based on the complementary functions method. For the suggested model, a finite element analysis computer program is coded in Fortran. Results of the presented method are compared with those obtained from ANSYS finite element software which uses the Newmark time integration method to solve the equations of motion. The governing equations of motion of the problem are first obtained in the time domain. Laplace transform is then applied and the set of simultaneous linear algebraic equations are solved by CFM in the Laplace domain for a set of Laplace parameters. The solutions obtained are transformed to the time domain using the modified Durbin's inverse numerical Laplace transform method.

The accuracy of the results of Newmark method (ANSYS - finite element software) depends on the appropriate selection of the optimum time increment. By using the presented method, highly accurate results can be obtained, even with a coarse time increment. It is manifest that combination of Laplace transform and Complementary Functions Method (CFM) is far more efficient than the conventional step-by-step integration methods. Laplace transformation gives a time-independent boundary-value problem in spatial coordinate which is then solved by CFM. The numerical examples has proved that the suggested procedure is highly accurate and efficient compared to various other numerical methods available in the literature and it can be easily applied to the planar curved rods.

\section{ACKNOWLEDGEMENTS}

The authors thank the Scientific Research Projects Directorate of Cukurova University for supporting the present study (FBA-2016-6007).

\section{REFERENCES}

[1] Haktanır, V. (1990). Investigation of the static, dynamic and buckling behavior of cylindrical helical rods by transfer matrix method. PhD. Thesis, University of Cukurova, Adana.

[2] Bayhan,S. (1993). Analysis of planner curved rods by stiffness matrix and transfer matrix method. MSc. Thesis, University of Cukurova, Adana.

[3] Bozkurt, M. (1995). The analysis of cylindrical vaults, circular and helicoidal structural systems by the complementary functions method-mathematica applications. MSc. Thesis, University of Cukurova, Adana.

[4] Yıldırım, V., İnce, N., and Kıral, E. (1997) "Static Analysis of compound planar frames with members of linear and circular axes by stiffness matrix method. Journal of Engineering and Environmental Sciences, pp.137-148

[5] Çalım, F.F. (2003). Dynamic analysis of viscoelastic, anisotropic curved spatial rod systems. PhD. Thesis, University of Cukurova, Adana.

[6] Kıraç, M. (2007). Dynamic analysis of straight composite rods. MSc. Thesis, University of Mustafa Kemal, Hatay.

[7] Aktan, H. (2008). In-Plane dynamic analysis of circular beams. MSc. Thesis, Istanbul Technical University, Istanbul.

[8] Çoban, M. (2008). Out-Of-Plane dynamic analysis of curved beams using mixed finite element method. MSc. Thesis, Istanbul Technical University, Istanbul.

[9] Akkurt, F.G. (2011). Dynamic analysis of straight and circular rods on elastic foundation. MSc. Thesis, University of Mustafa Kemal, Hatay.

[10] Karaca, N. (2014). Static and dynamic analysis of planar rods by the transfer and stiffness matrix methods. MSc. Thesis, University of Mustafa Kemal, Hatay.

[11] Ecer, S., (2015) "Longitudinal vibration analysis of straight bars using mixed finite element method. MSc. Thesis, Istanbul Technical University, Istanbul.

[12] Durbin, F. (1974). Numerical inversion of laplace transforms: An efficient improvement to Dubner and Abate's Method. The Computer Journal, vol.17, no.4 pp. 371 - 376. Doi: 10.1093/comjnl/17.4.371

[13] Temel, B., Çalim, F.F. And Tütüncü, N. (2004). Quasi-Static and dynamic response of viscoelastic helical rods. Journal of Sound and Vibration, 271:921 - 935. Doi: 10.1016/S0022-460X(03)00760-0

[14] ANSYS, Inc Release 15.0 (2013), Canonsburg, PA 